\title{
Images - Isolated forearm soft tissue metastasis from bladder cancer
}

Cameron Gregory Ashe ${ }^{1}$; Scott Bagnell ${ }^{2}$; Samantha Gray ${ }^{3}$

${ }^{1} \mathrm{MD}$ candidate, Dalhousie University Faculty of Medicine; ${ }^{2}$ Department of Urology, Dalhousie University, Saint John Regional Hospital; ${ }^{3}$ Department of Oncology, Dalhousie University, Saint John Regional Hospital; St. John, NB, Canada

Cite as: Can Urol Assoc J 2018 August 30; Epub ahead of print. http://dx.doi.org/10.5489/cuaj.5341

Published online August 30, 2018

$* * *$

\section{Introduction}

Bladder cancer is the ninth most prevalent cancer in the world and most common malignancy involving the urinary system with 430000 cases diagnosed in 2012.(1) Urothelial cell carcinoma (UCC, also known as transitional cell carcinoma) is considered to be the predominant histological type of bladder cancer in the United States and Western Europe. While greater than $50 \%$ of UCC cases are non-muscle invasive with relatively good prognosis, soft tissue invasion and metastatic spread offer much worse outcomes.(2) The most common areas for metastatic spread of UCC are the lymph nodes, bones, lung, and liver. Advanced UCC has a poor prognosis with an overall five year survival of less than 15\%.(3)

Recent reports of the distribution of UCC metastases from clinical sites found evidence of soft tissue metastases, however these occurred in the presence of advanced stage disease with lesions present in additional locations of the body. $(4,5)$ UCC metastases to soft tissue in the absence of disease elsewhere in the body are exceedingly rare and few cases are described.

We present the rare case of a 70-year-old patient with metastases to the soft tissue of the arm without other sites of recurrence, six months after radical cystoprostatectomy for muscle invasive UCC of the bladder.

\section{Case report}

A 70-year-old man presented to his general practitioner with gross hematuria. He underwent abdominal imaging, which showed a $6 \mathrm{~cm}$ exophytic bladder mass. Subsequent cystoscopy and transurethral resection of the bladder (TURB) confirmed a high grade urothelial cancer with muscle invasion.

He underwent four cycles of cisplatin-based neoadjuvant chemotherapy (delivered by peripherally inserted central catheter (PICC) in the right arm) followed by radical 
cystoprostatectomy. The final pathology confirmed a ypT2bN0 high grade urothelial cell carcinoma.

Subsequent imaging performed three months post-operatively demonstrated no evidence of local recurrence or metastatic disease.

The patient then presented to his family physician with left arm swelling five months post-operatively. Given the patient was on anticoagulation, and had a history of mild trauma, it was initially felt to be a hematoma, but a MRI was ordered to investigate.

The MRI of the arm (Fig. 1) found a soft tissue mass 3.0x2.2x1.5 cm in size. It was thought that this would be a very unusual metastasis from a primary bladder cancer and so the clinical opinion was suspicious of a sarcoma. A CT of the chest, abdomen and pelvis with enhancement done at the same time showed no evidence of metastatic disease from the bladder cancer.

The patient underwent an open biopsy which revealed a neoplastic infiltrate within the connective tissue stroma with foci of lymphovascular invasion (Fig. 2). The malignant cells were similar in appearance to those noted in the patient's previous cystectomy specimen. Immunostaining supported the histological interpretation of metastatic urothelial carcinoma.

A subsequent PET scan was arranged and no evidence of metastatic disease was found beyond the known left forearm lesion.

After discussion at multi-disciplinary rounds, the patient was referred for consideration of stereotactic body radiation therapy (SBRT) to the forearm metastasis. Unfortunately, given the proximity to skin, it was not recommended, and as such, palliative high dose radiation was arranged to the left forearm.

Two months subsequent to the radiation therapy, the mass persisted, with no real regression in the size of the mass, although there was noted to be central necrosis, and edema, likely radiation induced. Given no progression outside of the arm, after multidisciplinary consultation and discussion with the patient, the decision was made to pursue a more aggressive approach, and the patient underwent an attempted local excision of the tumor. However, damage to the tumour area was extensive and a resulting amputation of the left forearm and hand was deemed to be necessary and was performed.

Pathology of the specimen confirmed metastatic urothelial carcinoma involving soft tissue reaching the deep skeletal muscle and was histologically a match to previous specimens collected from the patient.

The patient is recovering well and to date (twelve months since the mass in the forearm was noted on MRI) no evidence of disease recurrence has been found.

\section{Discussion}

Soft tissue metastases from a distant primary lesion are rare in most cancers even with the large amount of soft tissue in the body. In the context of UCC, soft tissue metastases appear to be even more rare with recent reviews finding few cases of soft tissue metastases. (4-6) In addition, the 
metastases were found in instances of advanced stage cancer. Only one case study was found which also showed an isolated metastasis from UCC of the bladder to the sartorius muscle. (8) To our knowledge there has been no other UCC soft tissue metastases that has occurred in the presence of negative imaging studies, which demonstrate no other disease recurrence.

Most cases in the literature presented with localized and painful muscular swelling in a similar manner to our patient (6). Interestingly, as occurred in our patient, local trauma has been reported to be a risk factor potentially for development of metastases. It is hypothesized that injury may in some way alter the physiology and microenvironment at the site of trauma and therefore result in increased susceptibility to the development of metastatic disease at that site of trauma. $(7,8)$

The treatment for soft tissue metastases is unclear, and generally is palliative given most patients present in the context of advanced disease. Given the poor prognosis associated with metastatic bladder cancer, it is unknown whether aggressive treatment to an isolated metastasis is warranted. Past case studies of isolated metastases to soft tissue of have been treated aggressively with a variable combination of bloc excision, radiation and chemotherapy. $(9,10)$

Our patient will be followed closely for regular oncologic surveillance for recurrent metastatic disease.

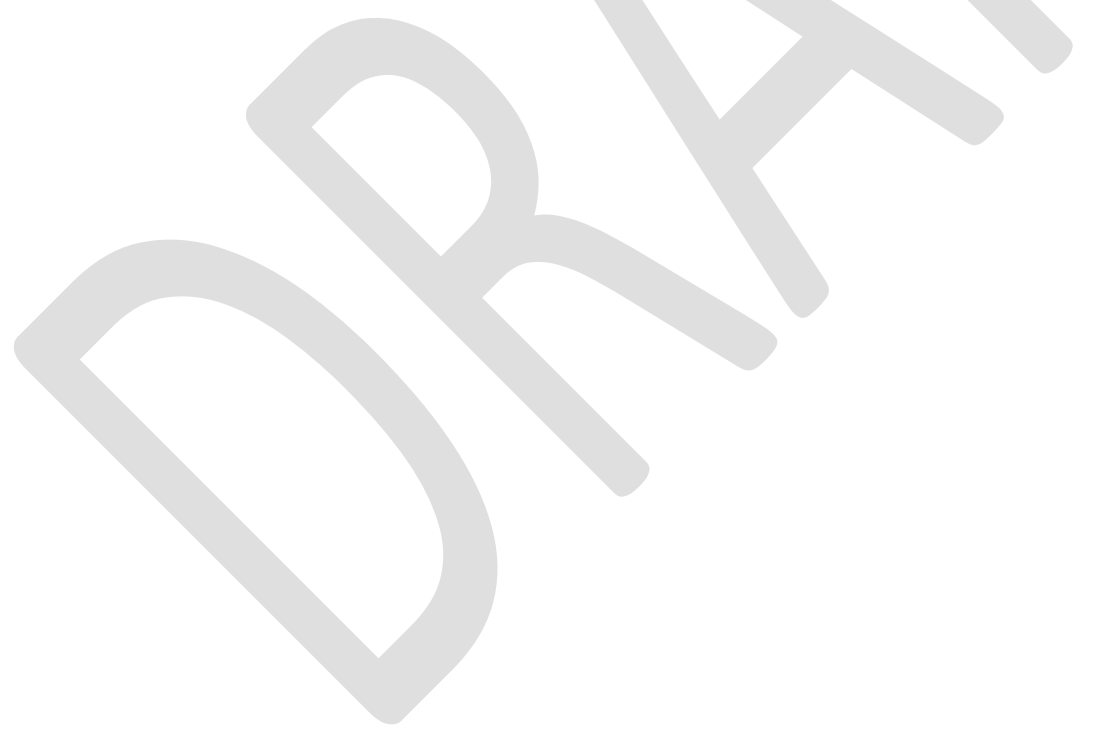




\section{References}

1. Torre LA, Bray F, Siegel RL, et al. Global cancer statistics, 2012. CA: a cancer journal for clinicians 2015;65:87-108.

2. Siegel RL, Miller KD, Jemal A. Cancer statistics, 2016. CA: a cancer journal for clinicians 2016;66:7-30.

3. von der Maase H, Sengelov L, Roberts JT, et al. Long-term survival results of a randomized trial comparing gemcitabine plus cisplatin, with methotrexate, vinblastine, doxorubicin, plus cisplatin in patients with bladder cancer. Journal of clinical oncology : official journal of the American Society of Clinical Oncology 2005;23:4602-8.

4. Shinagare AB, Ramaiya NH, Jagannathan JP, et al. Metastatic pattern of bladder cancer: correlation with the characteristics of the primary tumor. AJR American journal of roentgenology 2011;196:117-22.

5. Plaza JA, Perez-Montiel D, Mayerson J, et al. Metastases to soft tissue. Cancer 2008;112:193-203.

6. Guidi M, Fusetti C, Lucchina S. Skeletal Muscle Metastases to the Flexor Digitorum Superficialis and Profundus from Urothelial Cell Carcinoma and Review of the Literature. Case reports in urology 2016;2016:2387501.

7. Magee T, Rosenthal H. Skeletal muscle metastases at sites of documented trauma. AJR American journal of roentgenology 2002;178:985-8.

8. Bissell MJ, Hines WC. Why don't we get more cancer? A proposed role of the microenvironment in restraining cancer progression. Nature medicine 2011;17:320-9.

9. Katafigiotis I, Athanasiou A, Levis PK, et al. Metastasis to sartorius muscle from a muscle invasive bladder cancer. Case reports in medicine 2014;2014:524757.

10. Klune JR, Zuckerbraun B, Tsung A. Isolated skeletal muscle metastasis following successful treatment of laryngeal cancer: case report. International Seminars in Surgical Oncology : ISSO 2010;7:1-. 


\section{Figures and Tables}

Fig. 1. Imaging of left arm mass. $T 1$ fat saturated image from magnetic resonance imaging demonstrating a $3.0 \times 2.2 \times 1.5 \mathrm{~cm}$ lesion within the connective tissue stroma.

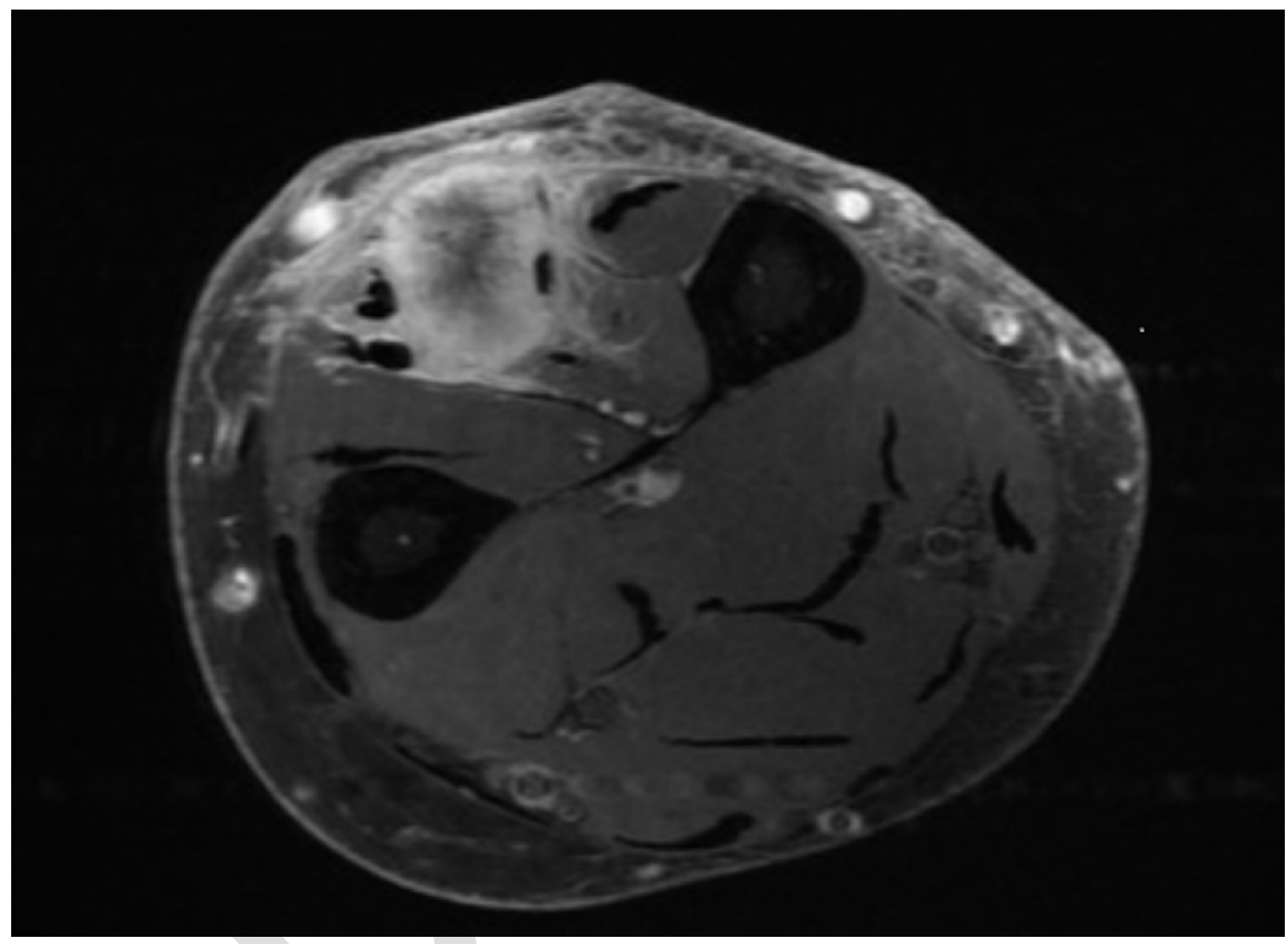


Fig. 2. Neoplastic infiltrate within the connective tissue stroma of the forearm with foci of lymphovascular invasion. Malignant cells showing (A) GATA 3 expression; and (B) CK20 positivity.
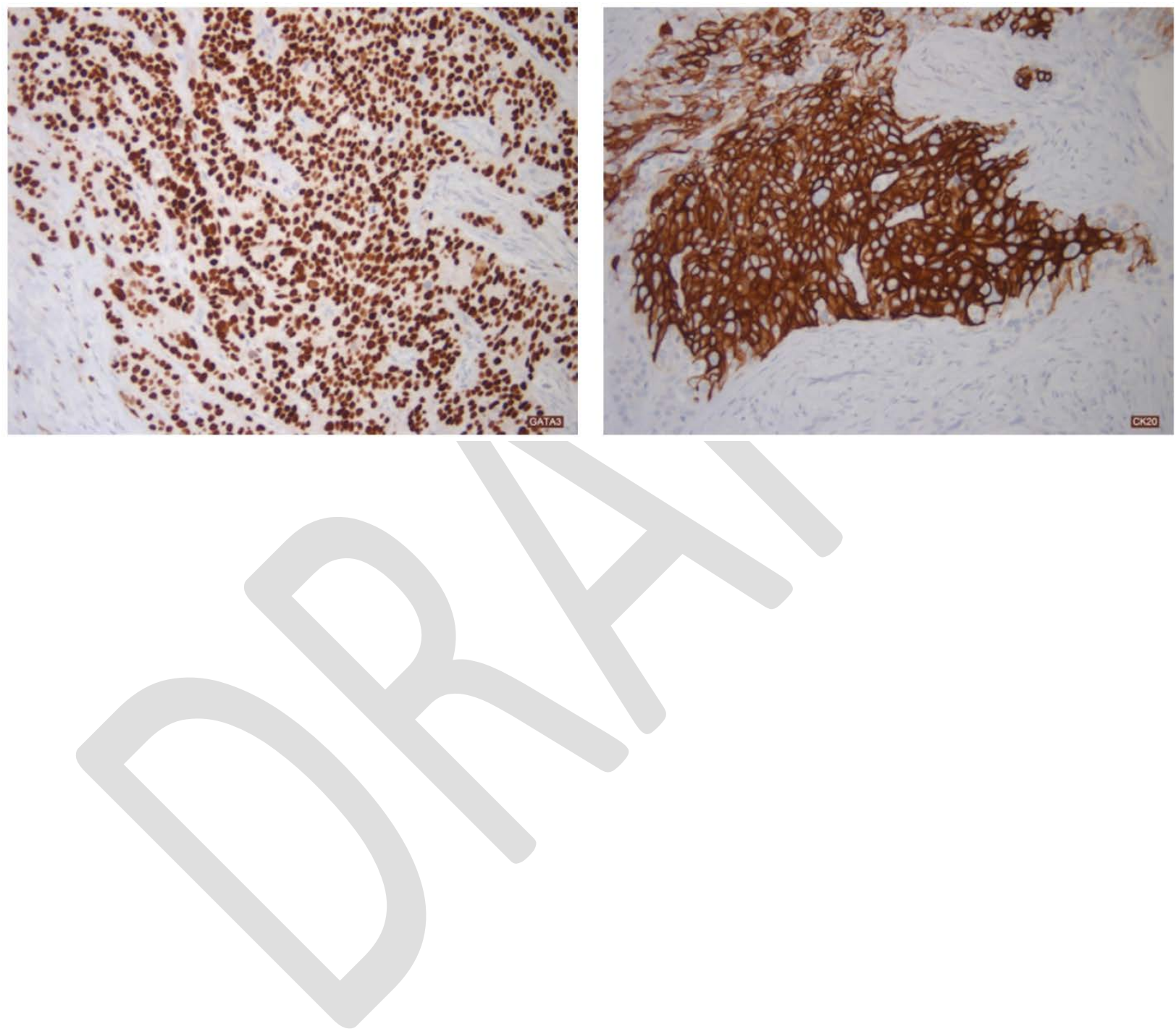